\title{
Atherogenic index of plasma predicts cerebrovascular accident occurrence in antineutrophil cytoplasmic antibody- associated vasculitis
}

Sung Soo Ahn' ${ }^{1}$, Lucy Eunju Lee ${ }^{1}$, Jung Yoon Pyo ${ }^{1}$, Jason Jungsik Song ${ }^{1,2}$, Yong-Beom Park ${ }^{1,2}$ and Sang-Won Lee L, $^{1,2^{*}}$

\begin{abstract}
Background: To investigate whether atherogenic index of plasma (AIP) at diagnosis is associated with the occurrence of cerebrovascular accident (CVA) or coronary artery disease (CAD) in antineutrophil cytoplasmic antibody-associated vasculitis (AAV).
\end{abstract}

Methods: The medical records of 167 AAV patients on initial diagnosis was reviewed, and 300 healthy controls were included. AIP was calculated using the following equation: AIP $=$ Log (triglyceride $[\mathrm{mg} / \mathrm{dL}] /$ high-density lipoprotein cholesterol [mg/dL]). AAV patients were divided into two groups according to the AIP cut-off of 0.11 . The event of stroke, transient ischemic attack, and cerebral hemorrhage was recorded as CVA, and CAD events consisted of either myocardial infarction and angina pectoris. CVA- and CAD- free survival rate between those with AIP $\geq 0.11$ and $<0.11$ were compared by the Kaplan-Meier analysis, and Cox hazard analysis was conducted to identify predictors of CVA.

Results: The median age of AAV patients were 59.0 years, and 54 (32.3\%) patients were male. One-hundred and fifteen (68.9\%) patients had AIP $<0.11$ and 52 (31.1\%) had AIP $\geq 0.11$. The mean Birmingham vasculitis activity score in AAV patients with AIP $<0.11$ was lower than that seen in patients with AIP $\geq 0.11$ (12.0 vs. 14.0, $P=0.041$ ). AAV patients had a significantly higher AIP compared to controls (mean -0.01 vs. $-0.10, P<0.001$ ). During follow-up, the occurrence of CVA and CAD was observed in 16 (9.6\%) and 14 (8.4\%) patients, respectively. In Kaplan-Meier analysis, AAV patients with AIP $\geq 0.11$ had significantly lower CVA-free survival rates than in those with AIP $<0.11(P=0.027)$, whereas there was no difference in CAD according to AIP $(P=0.390)$. Multivariable Cox analysis indicated that AIP $\geq 0.11$ at diagnosis was the sole predictor of CVA (Hazard ratio 3.392, 95\% confidence interval 1.076, 10.696, $P=$ $0.037)$.

(Continued on next page)

\footnotetext{
* Correspondence: sangwonlee@yuhs.ac

'Division of Rheumatology, Department of Internal Medicine, Yonsei University College of Medicine, 50-1 Yonsei-ro, Seodaemun-gu, Seoul, Republic of Korea 03722

${ }^{2}$ Institute for Immunology and Immunological Diseases, Yonsei University

College of Medicine, Seoul, Republic of Korea
}

C C The Author(s). 2020 Open Access This article is licensed under a Creative Commons Attribution 4.0 International License, which permits use, sharing, adaptation, distribution and reproduction in any medium or format, as long as you give appropriate credit to the original author(s) and the source, provide a link to the Creative Commons licence, and indicate if changes were made. The images or other third party material in this article are included in the article's Creative Commons licence, unless indicated otherwise in a credit line to the material. If material is not included in the article's Creative Commons licence and your intended use is not permitted by statutory regulation or exceeds the permitted use, you will need to obtain permission directly from the copyright holder. To view a copy of this licence, visit http://creativecommons.org/licenses/by/4.0/. The Creative Commons Public Domain Dedication waiver (http://creativecommons.org/publicdomain/zero/1.0/) applies to the data made available in this article, unless otherwise stated in a credit line to the data. 
(Continued from previous page)

Conclusions: AIP is significantly higher in AAV patients than in healthy controls, and AIP $\geq 0.11$ at diagnosis is a significant predictor of CVA during follow-up. Stringent surveillance should be provided in AAV patients with AIP $\geq$ 0.11 regarding the occurrence of CVA.

Trial registration: Retrospectively registered (4-2017-0673).

Keywords: Antineutrophil cytoplasmic antibody, Vasculitis, Atherogenic index of plasma, Cerebrovascular accident, Predictor

\section{Background}

Antineutrophil cytoplasmic antibody (ANCA)-associated vasculitis (AAV) is a chronic inflammatory disorder (CID) that usually involves the small-sized vasculatures and has three distinct subtypes: microscopic polyangiitis (MPA), granulomatosis with polyangiitis, and eosinophilic granulomatosis with polyangiitis $[1,2]$. Generally, AAV involves the vessels and induces necrotizing vasculitis within the arterioles, venules, and capillaries and could present with a wide spectrum of clinical manifestations [3]. Even though the pathogenesis of AAV is thought to be complex, it is now being increasingly understood that a breach of cellular and humoral immunity is responsible for the loss of self-tolerance, leading to inflammation and organ injury [4]. In addition, the overproduction of pro-inflammatory cytokines, such as tumor necrosis factor (TNF)- $\alpha$, interferon-gamma, interleukin (IL)-1b, IL-6, IL-8, and IL-18, is responsible for amplifying the vicious loop [5].

Chronic inflammation is generally associated with deregulated lipid metabolism skewed towards an atherogenic profile, and it is typically characterized by an increase of triglyceride (TG) and decrease of high-density lipoprotein (HDL)-cholesterol $[6,7]$. Even though multiple factors have been suggested regarding this phenomenon, the secretion of multiple inflammatory cytokines, i.e. TNF, IL-1, IL-2, and IL-6, is reported to be linked to the elevation of TG level, which is induced by accelerated lipolysis in the adipose tissue and the synthesis of fatty acids in the liver, while inhibiting hepatic fatty acid oxidation [8]. On the other hand, proinflammatory cytokines are also implicated in the decrease of HDL-cholesterol as a consequence of impaired production of apolipoprotein A-1, which is a major protein constituting the HDL-cholesterol [9]. Moreover, diminished formation of cholesterol ester, structural and functional alteration of HDL-cholesterol, and increased HDL-cholesterol clearance have been also thought be relevant to the decreased HDL-cholesterol level in the presence of chronic inflammation [7].

Emerging evidences now clearly indicate that patients with CID are prone to cardiovascular diseases (CVD) such as cerebrovascular accident (CVA), coronary artery disease (CAD), and thromboembolic diseases [10-12]. Notably, atherogenic index of plasma (AIP), which is calculated based on serum TG and HDL-cholesterol, is one of the laboratory indices indicating atherogenic status, and this has been used to assess the extent of dyslipidemia and predict the potential of developing CVA and CAD in various medical conditions [13-15]. Given that patients with CID are more often affected by CVDs than in healthy subjects and that increase of TG and decrease of HDL-cholesterol is present in those with chronic inflammation, it is possible that AIP is elevated in AAV patients and is associated with CVA and CAD. However, there have been no studies that determined the predictive potential of AIP regarding CVA and CAD events in AAV. Hence, this study investigated whether AIP in patients with AAV is higher than that in controls and to check whether this is associated the occurrence of CVA and CAD.

\section{Methods}

\section{Patient inclusion}

The medical records of 216 AAV patients when the initial diagnosis was made, were retrospectively reviewed. Patients were classified as AAV at the Division of Rheumatology in Severance Hospital, during the period from October 2000 to December 2019, based on the 2007 European Medicines Agency algorithm and the 2012 Chapel Hill Consensus Conferences definitions [1, 16]. The patients had well-documented medical records with accessible clinical and laboratory results, including Birmingham vasculitis activity score (BVAS) and fivefactor score (FFS) $[17,18]$. ANCA was detected using indirect immunofluorescence assay and antigen-specific enzyme-linked immunosorbent assays for myeloperoxidase and proteinase 3 . When the patients did not have ANCAs detected, the diagnosis of ANCA-negative vasculitis was made based on the clinical features and/or the histologic findings. All study subjects were followed up for at least 3 months after the diagnosis of AAV. At the time of diagnosis, patients were not on immunosuppressive agents and were not on medications to treat dyslipidemia. Further characteristics that would lead to a false-positive ANCA result, such as coexisting 
malignancies and serious infections, were also considered as exclusion criteria. Of the 216 AAV patients, 37 were excluded because TG and HDL-cholesterol levels were not assessed. Furthermore, 12 patients were currently taking drugs to treat dyslipidemia. Finally, 167 patients were included and analyzed in this study. In addition, body mass index (BMI) and lipid levels of TG, HDL-cholesterol, and low-density lipoprotein (LDL)cholesterol from 300 age- and gender-matched healthy controls, who had visited the healthcare center in Severance Hospital for a routine health examination and were not on medications to treat dyslipidemia, were assessed. The Institutional Review Board of Severance Hospital approved this study and was performed according to the ethical guidelines set forth in the Declaration of Helsinki (4-2017-0673).

\section{Baseline data and the definition of clinical outcomes}

Patients' demographic data such as age, gender, BMI, smoking history, and AAV subtypes were collected. ANCA positivity as well as AAV-specific indices of BVAS and FFS were obtained. Clinical manifestations and the presence of comorbidities, such as chronic kidney disease (stage III-V), diabetes mellitus, hypertension, and interstitial lung disease, were also assessed. In addition, the laboratory results including acute phase reactants of erythrocyte sedimentation rate (ESR) and Creactive protein (CRP) level, as well as AIP-related variables - serum total cholesterol, TG, HDL-cholesterol, and LDL-cholesterol levels - were reviewed. The clinical outcomes evaluated during the follow-up consisted of all-cause mortality, CVA, and CAD. In this study, we defined all-cause mortality as death regardless of the cause. We included events of cerebral infarction, transient ischemic attack, and cerebral hemorrhage as CVA and included acute coronary syndrome, including myocardial infarct and angina pectoris, as CAD. The followup duration was defined as the duration between the date of AAV diagnosis until the occurrence of clinical outcomes or the last visit, when subjects did not have an event corresponding to clinical outcomes.

\section{Calculation of AIP}

AIP was calculated using the following equation: AIP = Log (TG $(\mathrm{mg} / \mathrm{dL})$ / HDL-cholesterol $(\mathrm{mg} / \mathrm{dL}))$, as described previously [13]. Based on the results from previous studies, patients could be divided into three categories based on their AIP value. An AIP $<0.11$ as low risk, AIP of $0.11-0.21$ as intermediate risk, and AIP $>0.21$ as high risk $[19,20]$. In this study, AAV patients were divided into two groups according to AIP as follows: AAV patients with $\operatorname{AIP}<0.11(N=115)$ and AAV patients with AIP $\geq 0.11(N=52)$.

\section{Statistical analyses}

Continuous variables that were normally distributed are expressed as mean (standard deviation) and as median (interquartile range) when non-normally distributed. Categorical variables are expressed as number and the percentage. Significant differences between continuous variables were assessed using Student's t-test, MannWhitney $\mathrm{U}$ test, and analysis of variance, whereas differences between the categorical variables were analyzed by the Chi-square and Fisher's exact tests, as appropriate. Associations and the correlation coefficient between continuous variables were derived using the Pearson correlation analysis or Spearman correlation analysis. Comparison of the clinical outcome free survivals rates were analyzed using the Kaplan-Meier analysis with the log-rank test. Multivariable Cox hazards analysis was carried out using variables that showed statistical significance in the univariable analysis to identify predictors of CVA by the forward entry method. A $P$-value $<0.05$ was considered statistically significant. All statistical analyses were conducted using SPSS software version 23 for Windows (IBM Corp., Armonk, NY, USA).

\section{Results}

\section{Clinical characteristics of AAV patients at baseline}

Of the included 167 patients, the median age of the patients was 59 years, 54 (32.3\%) were male, and the mean BMI was $22.1 \mathrm{~kg} / \mathrm{m}^{2}$, respectively. The most common AAV subtype in the patients was MPA $(N=92,55.1 \%)$, and ANCA was detected in 133 patients (79.6\%). The most common clinical features were renal $(62.3 \%)$ and pulmonary (58.1\%) manifestations; hypertension as a comorbid condition was most frequently (53.3\%) observed. The laboratory test results are shown in Table 1 . The mean calculated AIP was - 0.01, and 115 (68.9\%) and 52 (31.1\%) patients were included in the AIP $<0.11$ and AIP $\geq 0.11$ group, respectively. Patients with AIP $\geq 0.11$ had a higher BVAS compared to those with AIP $<0.11$ $(P=0.041)$, even though there were no difference in FFS $(P=0.656)$. However, total serum protein level was significantly lower in patients with AIP $\geq 0.11$ than in those with AIP $<0.11(6.7 \mathrm{mg} / \mathrm{dL}$ vs. $6.4 \mathrm{mg} / \mathrm{dL}, P=0.043)$. As for AIP-related variables, TG level was higher in the AIP $\geq 0.11$ group than in the AIP $<0.11$ group, while the level of HDL-cholesterol was lower (both $P<0.001$ ) (Table 1).

On comparing demographic and AIP-related variables with healthy controls, AAV patients were found to have a significantly lower BMI $(P<0.001)$ and higher TG and HDL-cholesterol levels (median $113.0 \mathrm{mg} / \mathrm{dL}$ vs. 94.0 $\mathrm{mg} / \mathrm{dL}, P=0.002$ and median $51.0 \mathrm{mg} / \mathrm{dL}$ vs. $50.0 \mathrm{mg} / \mathrm{dL}$, $P=0.016)$. Moreover, AAV patients exhibited a significantly higher AIP value than controls (mean -0.01 vs. $-0.10, P<0.001)$ (Table 2). 
Table 1 Clinical characteristics of AAV patients at diagnosis

\begin{tabular}{|c|c|c|c|c|}
\hline Variables & $\begin{array}{l}\text { All AAV patients } \\
(\boldsymbol{N}=167)\end{array}$ & $\begin{array}{l}\text { AAV patients with AIP }<0.11 \\
(\boldsymbol{N}=115)\end{array}$ & $\begin{array}{l}\text { AAV patients with AIP } \geq 0.11 \\
(\boldsymbol{N}=52)\end{array}$ & $\boldsymbol{P}$-value \\
\hline \multicolumn{5}{|l|}{ Demographic data } \\
\hline Age (years) & $59.0(22.0)$ & $60.0(24.0)$ & $56.0(19.8)^{\mathrm{a}}$ & 0.369 \\
\hline Male gender $(\mathrm{N},(\%))$ & $54(32.3)$ & $34(29.6)$ & $20(38.5)$ & 0.255 \\
\hline Body mass index $\left(\mathrm{kg} / \mathrm{m}^{2}\right)$ & $22.1(4.5)^{\mathrm{a}}$ & $22.0(4.8)^{\mathrm{a}}$ & $23.2(4.3)^{\mathrm{a}}$ & 0.324 \\
\hline Smoking history (N, (\%)) & $6(3.6)$ & $3(2.6)$ & $3(5.8)$ & 0.310 \\
\hline AAV Subtypes (N, (\%)) & & & & 0.641 \\
\hline MPA & $92(55.1)$ & $64(55.7)$ & $28(53.8)$ & \\
\hline GPA & $38(22.8)$ & $24(20.9)$ & $14(26.9)$ & \\
\hline EGPA & $37(22.2)$ & $27(23.5)$ & $10(19.2)$ & \\
\hline \multicolumn{5}{|l|}{ ANCA positivity (N, (\%)) } \\
\hline MPO-ANCA (or P-ANCA) positivity & $114(68.3)$ & $79(68.7)$ & $35(67.3)$ & 0.858 \\
\hline PR3-ANCA (or C-ANCA) positivity & $27(16.2)$ & $16(13.9)$ & $11(21.2)$ & 0.239 \\
\hline Both ANCA positivity & $8(4.8)$ & $6(5.2)$ & $2(3.8)$ & 1.000 \\
\hline ANCA negativity & $34(20.4)$ & $26(22.6)$ & $8(15.4)$ & 0.283 \\
\hline \multicolumn{5}{|l|}{ AAV-specific indices } \\
\hline BVAS & $12.0(11.0)^{\mathrm{a}}$ & $12.0(11.0)^{\mathrm{a}}$ & $14.0(10.0)^{\mathrm{a}}$ & 0.041 \\
\hline FFS & $1.0(1.0)$ & $1.0(1.0)$ & $1.0(1.0)$ & 0.656 \\
\hline \multicolumn{5}{|l|}{ Clinical manifestations (N, (\%)) } \\
\hline General & $75(44.9)$ & $48(41.7)$ & $27(51.9)$ & 0.221 \\
\hline Cutaneous & $38(22.8)$ & $29(25.2)$ & $9(17.3)$ & 0.259 \\
\hline Muco-membranous /Ocular & $10(6.0)$ & $5(4.3)$ & $5(9.6)$ & 0.184 \\
\hline Ear nose throat & $75(44.9)$ & $51(44.3)$ & $24(46.2)$ & 0.828 \\
\hline Pulmonary & $97(58.1)$ & $69(60.0)$ & $28(53.8)$ & 0.455 \\
\hline Cardiovascular & $43(25.7)$ & $27(23.5)$ & $16(30.8)$ & 0.318 \\
\hline Gastrointestinal & $9(5.4)$ & $7(6.1)$ & $2(3.8)$ & 0.553 \\
\hline Renal & $104(62.3)$ & $69(60.0)$ & $35(67.3)$ & 0.367 \\
\hline Nervous & $56(33.5)$ & $38(33.0)$ & $18(34.6)$ & 0.842 \\
\hline \multicolumn{5}{|l|}{ Comorbidities (N, (\%)) } \\
\hline Chronic kidney disease (stage 3-5) & $53(31.7)$ & $33(28.7)$ & $20(38.5)$ & 0.209 \\
\hline Diabetes mellitus & $51(30.5)$ & $35(30.4)$ & $16(30.8)$ & 0.965 \\
\hline Hypertension & $89(53.3)$ & $57(49.6)$ & $32(61.5)$ & 0.151 \\
\hline Interstitial lung disease & $50(29.9)$ & $36(31.3)$ & $14(26.9)$ & 0.567 \\
\hline \multicolumn{5}{|l|}{ Laboratory results } \\
\hline White blood cell count $\left(/ \mathrm{mm}^{3}\right)$ & $9210.0(6347.5)$ & $9180.0(6430.0)$ & $10,230.0(6000.0)$ & 0.927 \\
\hline Hemoglobin (g/dL) & $11.3(2.3)^{\mathrm{a}}$ & $11.4(2.3)^{\mathrm{a}}$ & $11.0(2.5)^{\mathrm{a}}$ & 0.235 \\
\hline Platelet count $\left(\times 1000 / \mathrm{mm}^{3}\right)$ & $308.0(166.0)$ & $311.0(158.5)$ & $294.0(195.0)$ & 0.949 \\
\hline Fasting glucose (mg/dL) & $104.0(36.0)$ & $103.0(34.0)$ & $106.0(38.0)$ & 0.466 \\
\hline BUN (mg/dL) & $18.4(22.4)$ & $18.0(23.0)$ & $24.2(21.6)$ & 0.078 \\
\hline Creatinine (mg/dL) & $1.0(1.3)$ & $0.9(1.0)$ & $1.3(2.0)$ & 0.105 \\
\hline Total serum protein $(\mathrm{g} / \mathrm{dL})$ & $6.6(1.3)$ & $6.7(1.1)$ & $6.4(1.0)^{a}$ & 0.043 \\
\hline Serum albumin $(\mathrm{g} / \mathrm{dL})$ & $3.5(0.8)^{a}$ & $3.5(0.7)^{a}$ & $3.3(0.8)^{a}$ & 0.068 \\
\hline ALP (IU/L) & $72.0(38.0)$ & $69.0(36.0)$ & $77.0(51.0)$ & 0.339 \\
\hline AST (IU/L) & $18.5(9.0)$ & $19.0(8.0)$ & $17.0(10.0)$ & 0.121 \\
\hline
\end{tabular}


Table 1 Clinical characteristics of AAV patients at diagnosis (Continued)

\begin{tabular}{|c|c|c|c|c|}
\hline Variables & $\begin{array}{l}\text { All AAV patients } \\
(\boldsymbol{N}=167)\end{array}$ & $\begin{array}{l}\text { AAV patients with AIP }<0.11 \\
(\boldsymbol{N}=115)\end{array}$ & $\begin{array}{l}\text { AAV patients with AIP } \geq 0.11 \\
(\boldsymbol{N}=52)\end{array}$ & $\boldsymbol{P}$-value \\
\hline ALT (IU/L) & $16.5(14.8)$ & $15.0(13.5)$ & $19.0(18.0)$ & 0.985 \\
\hline Total bilirubin (mg/dL) & $0.5(0.3)$ & $0.5(0.3)$ & $0.5(0.4)$ & 0.620 \\
\hline $\mathrm{ESR}(\mathrm{mm} / \mathrm{hr})$ & $64.0(69.3)$ & $64.0(67.0)$ & $66.8(39.6)^{a}$ & 0.406 \\
\hline CRP (mg/L) & $17.0(85.8)$ & $15.0(69.3)$ & $25.0(119.3)$ & 0.123 \\
\hline \multicolumn{5}{|l|}{ AIP-related variables } \\
\hline Total cholesterol (mg/dL) & $178.0(58.0)$ & $182.1(45.8)^{\mathrm{a}}$ & $187.0(58.1)^{\mathrm{a}}$ & 0.561 \\
\hline $\mathrm{TG}(\mathrm{mg} / \mathrm{dL})$ & $113.0(73.0)$ & $97.5(35.5)^{\mathrm{a}}$ & $174.5(67.8)$ & $<0.001$ \\
\hline HDL-cholesterol (mg/dL) & $51.0(23.0)$ & $55.0(25.0)$ & $41.4(14.6)^{a}$ & $<0.001$ \\
\hline LDL-cholesterol (mg/dL) & $104.6(41.6)$ & $106.0(33.1)^{\mathrm{a}}$ & $102.2(45.1)$ & 0.917 \\
\hline AIP & $-0.01(0.2)^{\mathrm{a}}$ & $-0.1(0.3)$ & $0.3(0.1)^{a}$ & $<0.001$ \\
\hline
\end{tabular}

Values are expressed as a median (interquartile range) or $\mathrm{N}(\%)$

AAV ANCA-associated vasculitis, ANCA Antineutrophil cytoplasmic antibody, AIP Atherogenic index of plasma, MPA Microscopic polyangiitis, GPA Granulomatosis with polyangiitis, EGPA Eosinophilic GPA, MPO Myeloperoxidase, $P$ Perinuclear, PR3 Proteinase 3, C Cytoplasmic, BVAS Birmingham vasculitis activity score, FFS Fivefactor score, BUN Blood urea nitrogen, ALP Alkaline phosphatase, AST Aspartate aminotransferase, ALT Alanine aminotransferase, ESR Erythrocyte sedimentation rate, CRP C-reactive protein, TG Triglyceride, HDL High-density lipoprotein, LDL Low-density lipoprotein

${ }^{a}$ Normally distributed data are expressed as mean (standard deviation)

\section{Correlation of AIP with continuous variables}

At AAV diagnosis, AIP was found to be positively correlated with ESR $(r=0.171, P=0.028)$, CRP $(r=0.169, P=$ $0.030)$, and blood urea nitrogen $(r=0.187, P=0.016)$ and negatively correlated with total serum protein and serum albumin $(r=-0.201, P=0.010$ and $r=-0.209, P=$ 0.007). In addition, AIP was highly correlated with TG and HDL-cholesterol $(r=0.770, P<0.001$ and $r=-$ $0.579, P<0.001)$. AIP was not significantly correlated with age, BMI, BVAS, FFS, and the remaining laboratory variables (Table 3 ).

\section{Clinical outcomes and medications during follow-up}

During the follow-up period, 18 patients died, and 16 and 14 patients experienced CVA and CAD events,

Table 2 Comparison of demographic data and AIP-related variables between AAV patients and controls

\begin{tabular}{|c|c|c|c|}
\hline Variables & $\begin{array}{l}\text { AAV patients } \\
(\boldsymbol{N}=167)\end{array}$ & $\begin{array}{l}\text { Controls } \\
(\boldsymbol{N}=300)\end{array}$ & $\boldsymbol{P}$-value \\
\hline \multicolumn{4}{|l|}{ Demographic data } \\
\hline Age (years) & $59.0(22.0)$ & $58.0(21.0)$ & 0.382 \\
\hline Male gender $(\mathrm{N},(\%))$ & $54(32.3)$ & $82(27.3)$ & 0.254 \\
\hline Body mass index $\left(\mathrm{kg} / \mathrm{m}^{2}\right)$ & $22.3(3.1)^{\mathrm{a}}$ & $23.3(3.4)$ & $<0.001$ \\
\hline \multicolumn{4}{|l|}{ AIP-related variables } \\
\hline TG (mg/dL) & $113.0(73.0)$ & $94.0(60.0)$ & 0.002 \\
\hline HDL-cholesterol (mg/dL) & $51.0(23.0)$ & $50.0(19.0)$ & 0.016 \\
\hline LDL-cholesterol (mg/dL) & $104.6(41.6)$ & $106.0(48.0)$ & 0.211 \\
\hline AIP & $-0.01(0.2)^{a}$ & $-0.10(0.2)^{a}$ & $<0.001$ \\
\hline
\end{tabular}

Values are expressed as a median (interquartile range) or $\mathrm{N}$ (\%) AIP Atherogenic index of plasma, AAV ANCA-associated vasculitis, ANCA Antineutrophil cytoplasmic antibody, TG Triglyceride, HDL High-density lipoprotein, $L D L$ Low-density lipoprotein

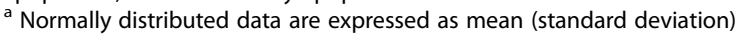

respectively. Concerning the medications used to treat AAV, glucocorticoids were most frequently administered $(N=155,92.8 \%)$, followed by cyclophosphamide $(N=87$, $52.1 \%)$ and azathioprine $(N=82,49.1 \%)$ (Table 4$)$.

\section{Comparison of clinical outcomes between AAV patients} with AIP $<0.11$ and those with AIP $\geq 0.11$

Regarding clinical outcomes, AAV patients with AIP $\geq$ 0.11 exhibited a significantly lower CVA-free survival rate than those with $\mathrm{AIP}<0.11 \quad(P=0.027)$. However, there were no significant differences regarding all-cause mortality and CAD between patients with AIP $<0.11$ and AIP $\geq 0.11(P=0.357$ and $P=0.390)$ (Fig. 1$)$.

\section{Cox hazards analysis for the prediction of CVA}

To investigate whether AIP $\geq 0.11$ at diagnosis could independently predict CVA occurrence during follow-up, we compared the predictive potential of conventional risk factors for CVA, ANCA types, AAV-specific indices, acute phase reactants, and AIP using the Cox hazards analysis. In the univariable analysis, both $\mathrm{AIP} \geq 0.11$ (Hazard ratio (HR) 3.391, 95\% confidence interval (CI) $1.075,10.695, P=0.037$ ) and CRP (HR 1.008, 95\% CI $1.001,1.016, P=0.035)$ were significantly associated with CVA during follow-up. In the multivariable analysis, only AIP $\geq 0.11$ at diagnosis was revealed to be significantly associated with CVA during follow-up (HR 3.392, 95\% CI 1.076, 10.696, $P=0.037$ ) (Table 5).

\section{Comparison of AIP according to gender, body mass index, age, and AAV subtypes}

To exclude the possibility of the influence of gender, BMI, age, and AAV subtypes in AIP, a subgroup analysis was performed. Male patients with AAV had 
Table 3 Relationship between AIP and continuous variables at diagnosis

\begin{tabular}{|c|c|c|}
\hline Variables & r-value & $\boldsymbol{P}$-value \\
\hline Age & 0.068 & 0.386 \\
\hline Body mass index ${ }^{a}$ & 0.102 & 0.191 \\
\hline White blood cell count $\left(/ \mathrm{mm}^{3}\right)$ & 0.080 & 0.309 \\
\hline Hemoglobin $(\mathrm{g} / \mathrm{dL})^{a}$ & -0.124 & 0.113 \\
\hline Platelet count $\left(\times 1000 / \mathrm{mm}^{3}\right)$ & 0.069 & 0.377 \\
\hline Fasting glucose (mg/dL) & 0.117 & 0.133 \\
\hline BUN (mg/dL) & 0.187 & 0.016 \\
\hline Creatinine $(\mathrm{mg} / \mathrm{dL})$ & 0.141 & 0.071 \\
\hline Total serum protein ( $\mathrm{g} / \mathrm{dL})$ & -0.201 & 0.010 \\
\hline Serum albumin $(\mathrm{g} / \mathrm{dL})^{\mathrm{a}}$ & -0.209 & 0.007 \\
\hline ALP (IU/L) & 0.100 & 0.199 \\
\hline AST (IU/L) & -0.093 & 0.237 \\
\hline ALT (IU/L) & -0.036 & 0.647 \\
\hline Total bilirubin (mg/dL) & 0.045 & 0.569 \\
\hline ESR (mm/hr) & 0.171 & 0.028 \\
\hline CRP (mg/L) & 0.169 & 0.030 \\
\hline Total cholesterol (mg/dL) & -0.075 & 0.338 \\
\hline $\mathrm{TG}(\mathrm{mg} / \mathrm{dL})$ & 0.770 & $<0.001$ \\
\hline HDL-cholesterol (mg/dL) & -0.579 & $<0.001$ \\
\hline LDL-cholesterol (mg/dL) & -0.035 & 0.650 \\
\hline $\mathrm{BVAS}^{\mathrm{a}}$ & 0.124 & 0.111 \\
\hline FFS & 0.093 & 0.232 \\
\hline
\end{tabular}

AIP Atherogenic index of plasma, BUN Blood urea nitrogen, ALP Alkaline phosphatase, AST Aspartate aminotransferase, ALT Alanine aminotransferase, ESR Erythrocyte sedimentation rate, CRP C-reactive protein, TG Triglyceride, $H D L$ High-density lipoprotein, LDL Low-density lipoprotein, BVAS Birmingham vasculitis activity score, FFS Five-factor score

${ }^{\text {a }}$ Normally distributed data

significantly higher AIP than female patients with AAV $(P=0.026)$. However, there were no significant differences in AIP regarding BMI (divided based on the Asian Pacific cut-off values) $(P=0.334)$ and age $(P=0.196)$ [21]. Furthermore, AIP was not found to differ based on AAV subtypes $(P=0.407)$ (Fig. 2$)$.

\section{Discussion}

In line with the knowledge that patients with CIDs are at increased risk of developing CVDs, several studies have demonstrated that the risk of CVDs is heightened in AAV patients. A long-term population-based study revealed that the risk of CVD and CVA in AAV patients were 3- and 8-fold higher than those in matched subjects [22]. In addition, a retrospective study that was performed in the United Kingdom has shown that AAV patients have high incidence of arterial and venous thrombosis [23]. Moreover, a meta-analysis by Houben et al. demonstrated that the risk of developing
Table 4 Outcomes and the medications administered in AAV patients during follow-up

\begin{tabular}{ll}
\hline AAV patients & Values \\
\hline Clinical outcomes during follow-up (N, (\%)) & $18(10.8)$ \\
All-cause mortality (N, (\%)) & $33.7(65.6)$ \\
Follow-up duration based on all-cause mortality (months) & $16(9.6)$ \\
CVA (N, (\%)) & $30.5(64.0)$ \\
Follow-up duration based on CVA (months) & $14(8.4)$ \\
CVD (N, (\%)) & $32.8(63.6)$ \\
Follow-up duration based on CVD (months) & $155(92.8)$ \\
Medications administered during follow-up (N, (\%)) & $87(52.1)$ \\
Glucocorticoid & $29(17.4)$ \\
Cyclophosphamide & $82(49.1)$ \\
Rituximab & $22(13.2)$ \\
Azathioprine & $11(6.6)$ \\
Mycophenolate mofetil & $12(7.2)$ \\
\hline Tacrolimus & \\
Methotrexate & \\
\hline Values are expressed as a median (interquartile range, IQR) or N (\%) \\
$\begin{array}{l}\text { AAV ANCA-associated vasculitis, ANCA Antineutrophil cytoplasmic antibody, } \\
\text { CVA Cerebrovascular accident, CVD Cardiovascular disease }\end{array}$
\end{tabular}

cardiovascular events was higher in AAV patients compared to the general population [24]. Therefore, it is clinically important to uncover predictors that could help estimate the development of CVDs in AAV patients, as this could be a potentially life-threatening event.

To the best of our knowledge, this is the first study that evaluated the clinical effectiveness of using AIP to predict CVA and CVD in AAV patients. Consistent with what was initially expected, the observations from this study demonstrated that AAV patients had significantly higher AIP compared to healthy controls ( 0.01 vs. -0.12 , $P<0.001$ ). Moreover, AAV patients with AIP $\geq 0.11$ on diagnosis exhibited significantly higher disease activity at baseline, and the occurrence of CVA events during the follow-up was more frequent compared to those with AIP $<0.11$. In addition, Cox hazards analysis revealed that $\mathrm{AIP} \geq 0.11$ at diagnosis is an independent predictor for CVA during follow-up, even when various conventional risk factors for CVA, ANCA types, AAV-specific indices, and acute phase reactants were taken into consideration [17, 18, 25-28]. On the basis of the results of this study, it could be suggested that the occurrence of CVA should be actively monitored in AAV patients, especially in those with AIP $\geq 0.11$, when the initial diagnosis is established.

In the present study, the cut-off value of AIP $\geq 0.11$ was adopted to predict de novo CVA events in AAV patients; this was done since the number of patients with 

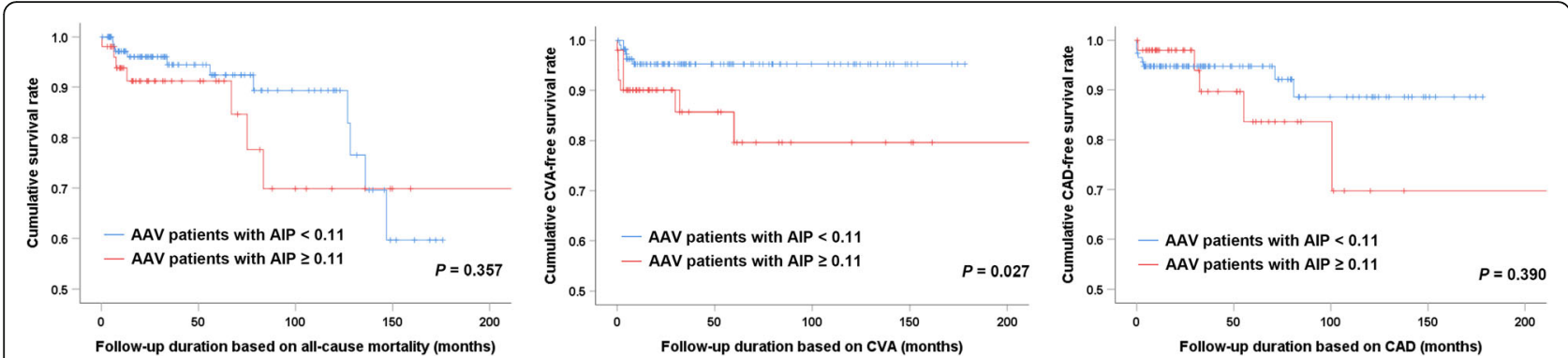

Fig. 1 Comparison of the cumulative clinical outcome free survival rate in patients with AIP $<0.11$ and AIP $\geq 0.11$. Among the clinical outcomes, AAV patients with AIP $\geq 0.11$ exhibited a significantly lower cumulative CVA-free survival rate than those with AIP $<0.11$, while there was no difference regarding all-cause mortality and CAD. AIP: atherogenic index of plasma; AAV: ANCA-associated vasculitis; ANCA: antineutrophil cytoplasmic antibody; CVA: cerebrovascular accident; CAD: coronary artery disease

AIP $0.11-0.21(N=20)$ and AIP $>0.21$ was small $(N=$ 32 ) and since the events of CVA and CAD were observed in a relatively small number of patients. Therefore, categorizing patients into the three different groups of AIP $<0.11$, AIP $0.11-0.21$, and AIP $>0.21$ could result in a false negative result owing to a low statistical power. Indeed, when the clinical outcomes were compared by dividing the patients into three groups, the patients with AIP $<0.11$ were less likely to experience CVA compared to those with AIP $0.11-0.21$ and AIP $>0.21$, even though the risk of developing CVA was not directly incremental (Fig. 3).

Previous studies have demonstrated a variable range of AIPs, and variations could arise because of differences in ethnicity, gender, geographical factors, and the medical conditions investigated. A study by Zhu et al. has shown that the mean AIP values in Chinese people with and without obesity were 0.13 and -0.04 [29]. On the other

Table 5 Predictors for the occurrence of CVA during follow-up

\begin{tabular}{|c|c|c|c|c|c|c|}
\hline \multirow[t]{2}{*}{ Variables } & \multicolumn{3}{|c|}{ Univariable } & \multicolumn{3}{|c|}{ Multivariable } \\
\hline & $\mathrm{HR}$ & $95 \% \mathrm{Cl}$ & $\boldsymbol{P}$-value & $\mathrm{HR}$ & $95 \% \mathrm{Cl}$ & $\boldsymbol{P}$-value \\
\hline \multicolumn{7}{|l|}{ Demographic data } \\
\hline Age (years) & 1.027 & $0.982,1.074$ & 0.243 & & & \\
\hline Male gender & 0.862 & $0.259,2.874$ & 0.809 & & & \\
\hline Body mass index $\left(\mathrm{kg} / \mathrm{m}^{2}\right)$ & 0.991 & $0.826,1.190$ & 0.924 & & & \\
\hline Smoking history & 0.047 & $0.000,32,787.590$ & 0.656 & & & \\
\hline \multicolumn{7}{|l|}{ Comorbidities } \\
\hline Chronic kidney disease (stage 3-5) & 1.069 & $0.322,3.551$ & 0.914 & & & \\
\hline Diabetes mellitus & 1.527 & $0.484,4.810$ & 0.470 & & & \\
\hline Hypertension & 1.636 & $0.492,5.442$ & 0.422 & & & \\
\hline \multicolumn{7}{|l|}{ ANCA positivity } \\
\hline MPO-ANCA (or P-ANCA) positivity & 0.678 & $0.215,2.142$ & 0.508 & & & \\
\hline PR3-ANCA (or C-ANCA) positivity & 1.041 & $0.228,4.760$ & 0.958 & & & \\
\hline \multicolumn{7}{|l|}{ AAV-specific indices } \\
\hline BVAS & 1.074 & $0.995,1.160$ & 0.066 & & & \\
\hline FFS & 1.476 & $0.851,2.559$ & 0.165 & & & \\
\hline \multicolumn{7}{|l|}{ Acute phase reactants } \\
\hline $\mathrm{ESR}(\mathrm{mm} / \mathrm{hr})$ & 1.001 & $0.986,1.017$ & 0.860 & & & \\
\hline CRP (mg/L) & 1.008 & $1.001,1.016$ & 0.035 & & & \\
\hline$A I P \geq 0.11$ & 3.391 & $1.075,10.695$ & 0.037 & 3.392 & $1.076,10.696$ & 0.037 \\
\hline
\end{tabular}

CVA Cerebrovascular accident, ANCA Antineutrophil cytoplasmic antibody, MPO Myeloperoxidase, P Perinuclear, PR3 Proteinase 3, C Cytoplasmic, BVAS Birmingham vasculitis activity score, FFS Five factor score, ESR Erythrocyte sedimentation rate, CRP C-reactive protein, AIP Atherogenic index of plasma 

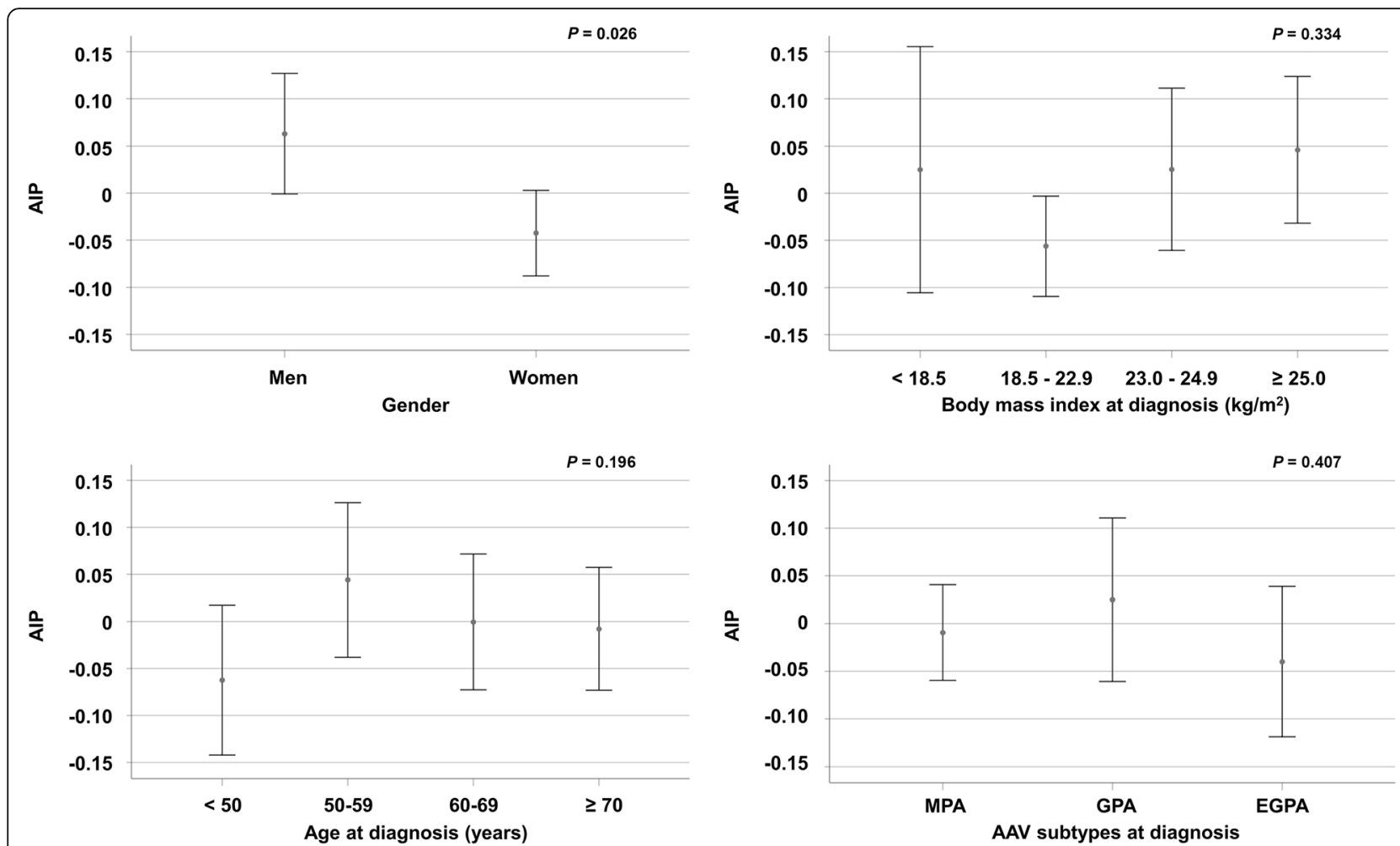

Fig. 2 Comparison of AIP based on gender, body mass index, age, and AAV subtypes. Male patients with AAV exhibited a higher AIP than female patients with AAV. However, no significant differences in AIP according to body mass index, age, and AAV subtypes were found. AIP: atherogenic index of plasma; AAV: ANCA-associated vasculitis; ANCA: antineutrophil cytoplasmic antibody; MPA: microscopic polyangiitis; GPA: granulomatosis with polyangiitis; EGPA: eosinophilic GPA

hand, Wu et al. identified the mean value of AIP in postmenopausal women with CAD and without CAD as 0.20 and 0.10 , respectively; furthermore, a study that estimated AIP in subjects undergoing chronic dialysis reported median values of AIP as 0.47 [13, 30]. Notably, several studies have been performed to evaluate AIP levels in CIDs, and a study that compared AIP levels in patients with SLE and controls showed that SLE patients had significantly higher AIP levels than controls, which is consistent with the result of this study [31]. Moreover, it was also described that AIP could be a predictor of subclinical atherosclerosis, particularly carotid artery intima-media thickness, in subjects with SLE, Behçet disease, and ankylosing spondylitis, emphasizing that subjects with higher AIP could be more vulnerable to CVA among patients with CIDs [31-33]. However, as most of the studies that were performed in patients with CIDs did not directly evaluate the incidence of CVAs, additional research is necessary to determine the relationship between AIP and CVAs in CIDs.

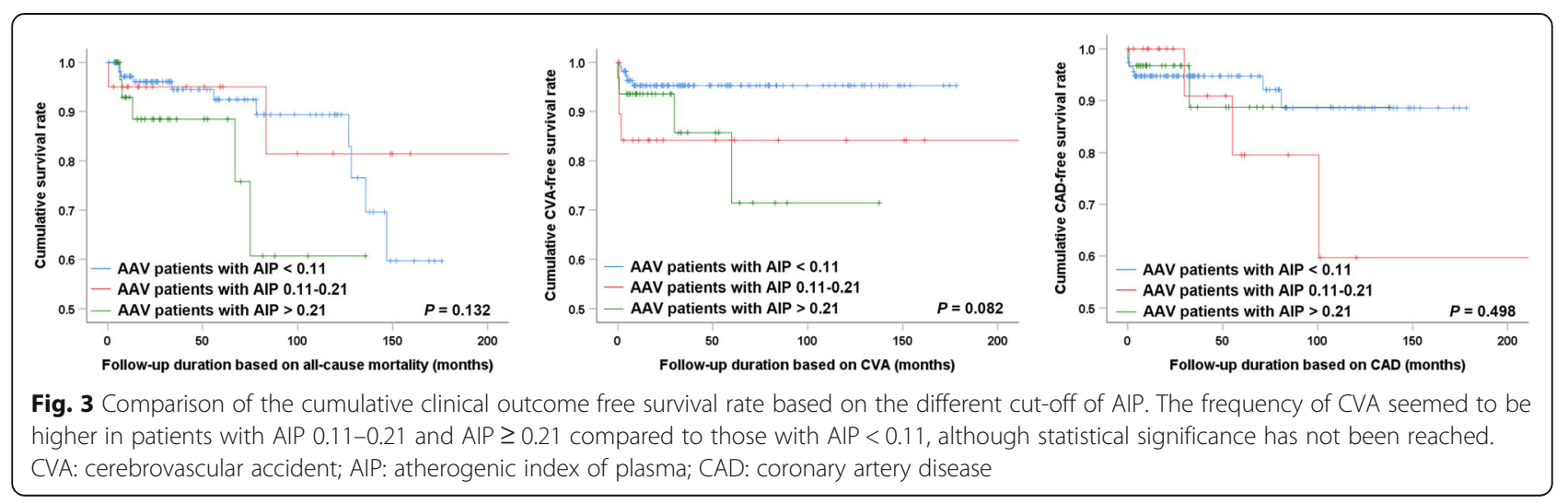


Besides AIP, previous data have proposed that higher cholesterol levels, such as TC, LDL-cholesterol, and TG could be used to predict the incidence of CVDs, even though inconsistent results were obtained across studies [34-37]. Accordingly, when the predictive value of different laboratory measures comprising AIP was investigated, AIP $\geq 0.11$ and HDL-cholesterol were found to be significantly associated with the incidence of CVA. However, given that AIP and HDL-cholesterol are closely associated because AIP level also includes the level of HDL-cholesterol in its calculation, further research is necessary to identify which is the most appropriate measure in estimating CVAs in AAV.

It has been acknowledged that higher disease activity is generally associated with increased risk of CVDs in patients with CIDs $[38,39]$. Accordingly, on comparing the baseline characteristics of patients with AIP $\geq 0.11$ and AIP $<0.11$, it was found that the difference of BVAS between the groups was significant $(P=0.041)$. Furthermore, in regard to the medications administered during follow-up, both cyclophosphamide $(63.5 \%$ vs. $47.0 \%, P=0.048)$ and rituximab (26.9\% vs. $13.0 \%, P=0.028$ ) were administered more commonly to AAV patients with AIP $\geq 0.11$ than to those with AIP $<0.11$ (Table 6). Based on the recommendations for the management of AAV, either cyclophosphamide or rituximab together with glucocorticoid should be given to AAV patients with lifethreatening disease [40]. Taken together, it can be speculated that the inflammatory burden in AAV patients with $\mathrm{AIP} \geq 0.11$ is significantly higher than that of patients with AIP $<0.11$ during follow-up, leading to an increased risk for CVA. In particular, it should be noted that the sum of the inflammatory burdens could not be simply estimated through BVAS, which consists of multiple measures that may not properly indicate the dynamic changes of vascular inflammation [41].

A previous study suggested that the major factors influencing AIP were gender, obesity, and older age
[42]. Accordingly, in this study, a subgroup analysis was performed to determine whether these factors affected the level of AIP. Of note, even though it was also revealed that male AAV patients exhibited significantly elevated AIP levels compared with females, which is similar to the previous studies, AIP was not found to differ based on BMI, age, and disease subtypes in the present study. Even though this discrepancy could be explained by the difference in the study population and the study design, it seems apparent that the impact of AIP in health and diseases could be variable and should be better investigated.

\section{Study strengths and limitations}

The most important strength of the present study was that it demonstrated, for the first time, that AIP at diagnosis is an independent predictor for CVA. However, several issues should be considered as limitations. First, the study was performed retrospectively and the clinical outcomes of the patients were identified by reviewing the hospital's medical records. In addition, the optimal cut-off value of AIP in predicting CVA could not be defined by this study. Second, because only laboratory data at initial diagnosis was used to calculate AIP, it is unclear whether dynamic changes in AIP levels might be more relevant to the risk of CVAs. Third, the precise mechanism of how AIP is associated with increased CVA events could not be elucidated. Future prospective studies with a larger number of patients will help verify the results of this study and provide more information regarding the potential of AIP in predicting CVA in AAV.

\section{Conclusions}

AIP in AAV patients was significantly higher than that in controls. Also, AIP $\geq 0.11$ at diagnosis could predict CVA occurrence during follow-up. These results suggest that stringent surveillance is required in AAV patients with AIP $\geq 0.11$ regarding the occurrence of CVA.

Table 6 Comparison of medication usage between patients with AIP $\geq 0.11$ and AIP $<0.11$ during follow-up

\begin{tabular}{|c|c|c|c|c|}
\hline & $\begin{array}{l}\text { All AAV patients } \\
(\boldsymbol{N}=167)\end{array}$ & $\begin{array}{l}\text { AAV patients with AIP }<0.11 \\
(\boldsymbol{N}=115)\end{array}$ & $\begin{array}{l}\text { AAV patients with AIP } \geq 0.11 \\
(\boldsymbol{N}=52)\end{array}$ & $\boldsymbol{P}$-value \\
\hline \multicolumn{5}{|c|}{ Medications administered during follow-up (N, (\%)) } \\
\hline Glucocorticoid & $155(92.8)$ & $104(90.4)$ & $51(98.1)$ & 0.107 \\
\hline Cyclophosphamide & $87(52.1)$ & $54(47.0)$ & $33(63.5)$ & 0.048 \\
\hline Rituximab & $29(17.4)$ & $15(13.0)$ & $14(26.9)$ & 0.028 \\
\hline Azathioprine & $82(49.1)$ & $52(45.2)$ & $30(57.7)$ & 0.135 \\
\hline Mycophenolate mofetil & $22(13.2)$ & $13(11.3)$ & $9(17.3)$ & 0.288 \\
\hline Tacrolimus & $11(6.6)$ & $6(5.2)$ & $5(9.6)$ & 0.289 \\
\hline Methotrexate & $12(7.2)$ & $10(8.7)$ & $2(3.8)$ & 0.345 \\
\hline
\end{tabular}

AIP Atherogenic index of plasma, AAV ANCA-associated vasculitis, ANCA Antineutrophil cytoplasmic antibody 


\section{Abbreviations}

AAV: Antineutrophil cytoplasmic antibody-associated vasculitis; AIP: Atherogenic index of plasma; ANCA: Antineutrophil cytoplasmic antibody; BMI: Body mass index; BVAS: Birmingham vasculitis activity score; CAD: Coronary artery disease; Cl: Confidence interval; CID: Chronic inflammatory disorder; CRP: C-reactive protein; CVA: Cerebrovascular accident; CVD: Cardiovascular disease; ESR: Erythrocyte sedimentation rate; FFS: Five-factor score; HDL: High-density lipoprotein; HR: Hazard ratio; IL: Interleukin; LDL: Low-density lipoprotein; MPA: Microscopic polyangiitis; TG: Triglyceride; TNF: Tumor necrosis factor

\section{Acknowledgements}

Not applicable.

\section{Authors' contributions}

SSA and SWL conceived the study. SSA, LEL, JYP, and SWL participated in the design and data collection. SSA performed statistical analyses, SSA, LEL, and SWL drafted the manuscript. JJS, YBP, and SWL edited and checked the manuscript. All of the authors have read and approved the final manuscript.

\section{Funding}

This work was supported by the National Research Foundation of Korea (NRF) grant funded by the Korean Government (MSIT) (NRF2018R1A5A2025079).

\section{Availability of data and materials}

The datasets used and/or analyzed during the current study are available from the corresponding author on reasonable request.

\section{Ethics approval and consent to participate}

This study was approved by the Institutional Review Board of Severance Hospital (4-2017-0673). The need for patients' written informed consent was waived, as this was a retrospective study.

\section{Consent for publication}

\section{Not applicable.}

\section{Competing interests}

The authors declare that they have no competing interests.

\section{Received: 26 May 2020 Accepted: 5 August 2020}

\section{Published online: 14 August 2020}

\section{References}

1. Jennette JC, Falk RJ, Bacon PA, Basu N, Cid MC, Ferrario F, et al. 2012 revised international Chapel Hill consensus conference nomenclature of vasculitides. Arthritis Rheum. 2013;65(1):1-11.

2. Hunter RW, Welsh N, Farrah TE, Gallacher PJ, Dhaun N. ANCA associated vasculitis. BMJ. 2020;369:m1070.

3. Yates M, Watts R. ANCA-associated vasculitis. Clin Med (Lond). 2017;17(1): 60-4.

4. Wilde B, van Paassen P, Witzke O, Tervaert JWC. New pathophysiological insights and treatment of ANCA-associated vasculitis. Kidney Int. 2011;79(6): 599-612.

5. Gómez-Puerta JA, Bosch X. Anti-neutrophil cytoplasmic antibody pathogenesis in small-vessel vasculitis: an update. Am J Pathol. 2009;175(5): $1790-8$

6. Hahn BH, Grossman J, Ansell BJ, Skaggs BJ, McMahon M. Altered lipoprotein metabolism in chronic inflammatory states: proinflammatory high-density lipoprotein and accelerated atherosclerosis in systemic lupus erythematosus and rheumatoid arthritis. Arthritis Res Ther. 2008;10(4):213.

7. Feingold KR, Grunfeld $C$. The effect of inflammation and infection on lipids and lipoproteins: MDText.com, Inc.; 2000. https://www.ncbi.nlm.nih.gov/ books/NBK326741/. Accessed 7 July 2020.

8. Khovidhunkit W, Kim MS, Memon RA, Shigenaga JK, Moser AH, Feingold KR, et al. Effects of infection and inflammation on lipid and lipoprotein metabolism: mechanisms and consequences to the host. J Lipid Res. 2004; 45(7):1169-96.

9. Haas MJ, Mooradian AD. Regulation of high-density lipoprotein by inflammatory cytokines: establishing links between immune dysfunction and cardiovascular disease. Diabetes Metab Res Rev. 2010;26(2):90-9.
10. Zeller CB, Appenzeller S. Cardiovascular disease in systemic lupus erythematosus: the role of traditional and lupus related risk factors. Curr Cardiol Rev. 2008;4(2):116-22.

11. DeMizio DJ, Geraldino-Pardilla LB. Autoimmunity and inflammation link to cardiovascular disease risk in rheumatoid arthritis. Rheumatol Ther. 2020;7(1): 19-33.

12. Manolis AS, Tzioufas AG. Cardio-rheumatology: cardiovascular complications in systemic autoimmune rheumatic diseases / is inflammation the common ink and target? Curr Vasc Pharmacol. 2020. https://doi.org/10.2174/ 1570161118666200514222236.

13. Wu TT, Gao Y, Zheng YY, Ma YT, Xie X. Atherogenic index of plasma (AIP): a novel predictive indicator for the coronary artery disease in postmenopausal women. Lipids Health Dis. 2018;17(1):197.

14. Cai G, Shi G, Xue S, Lu W. The atherogenic index of plasma is a strong and independent predictor for coronary artery disease in the Chinese Han population. Medicine (Baltimore). 2017;96(37):e8058.

15. Qin Z, Zhou K, Li Y, Cheng W, Wang Z, Wang J, et al. The atherogenic index of plasma plays an important role in predicting the prognosis of type 2 diabetic subjects undergoing percutaneous coronary intervention: results from an observational cohort study in China. Cardiovasc Diabetol. 2020; 19(1):23.

16. Watts R, Lane S, Hanslik T, Hauser T, Hellmich B, Koldingsnes W, et al. Development and validation of a consensus methodology for the classification of the ANCA-associated vasculitides and polyarteritis nodosa for epidemiological studies. Ann Rheum Dis. 2007;66(2):222-7.

17. Mukhtyar C, Lee R, Brown D, Carruthers D, Dasgupta B, Dubey S, et al. Modification and validation of the Birmingham vasculitis activity score (version 3). Ann Rheum Dis. 2009;68(12):1827-32.

18. Guillevin L, Pagnoux C, Seror R, Mahr A, Mouthon L, Le Toumelin P. The five-factor score revisited: assessment of prognoses of systemic necrotizing vasculitides based on the French vasculitis study group (FVSG) cohort. Medicine (Baltimore). 2011:90(1):19-27.

19. Wang Q, Zheng D, Liu J, Fang L, Li Q. Atherogenic index of plasma is a novel predictor of non-alcoholic fatty liver disease in obese participants: a cross-sectional study. Lipids Health Dis. 2018;17(1):284.

20. Akbas EM, Timuroglu A, Ozcicek A, Ozcicek F, Demirtas L, Gungor A, et al. Association of uric acid, atherogenic index of plasma and albuminuria in diabetes mellitus. Int J Clin Exp Med. 2014;7(12):5737-43.

21. Lee DH, Kim YS, Chae HS, Han K. Nationwide analysis of the relationships between mental health, body mass index and tinnitus in premenopausal female adults in Korea: 2010-2012 KNHANES. Sci Rep. 2018;8(1):7028.

22. Berti A, Matteson EL, Crowson CS, Specks U, Cornec D. Risk of cardiovascular disease and venous thromboembolism among patients with incident ANCA-associated vasculitis: a 20-year population-based cohort study. Mayo Clin Proc. 2018;93(5):597-606.

23. Kang A, Antonelou M, Wong NL, Tanna A, Arulkumaran N, Tam FWK, et al. High incidence of arterial and venous thrombosis in antineutrophil cytoplasmic antibody-associated vasculitis. J Rheumatol. 2019:46(3):285-93.

24. Houben E, Penne EL, Voskuyl AE, van der Heijden JW, Otten RHJ, Boers M, et al. Cardiovascular events in anti-neutrophil cytoplasmic antibodyassociated vasculitis: a meta-analysis of observational studies. Rheumatology (Oxford). 2018:57(3):555-62.

25. Murray CJ, Atkinson C, Bhalla K, Birbeck G, Burstein R, Chou D, et al. The state of US health, 1990-2010: burden of diseases, injuries, and risk factors. JAMA. 2013;310(6):591-608.

26. Boehme AK, Esenwa C, Elkind MS. Stroke risk factors, genetics, and prevention. Circ Res. 2017;120(3):472-95.

27. McAdoo SP, Medjeral-Thomas N, Gopaluni S, Tanna A, Mansfield N, Galliford J, et al. Long-term follow-up of a combined rituximab and cyclophosphamide regimen in renal anti-neutrophil cytoplasm antibodyassociated vasculitis. Nephrol Dial Transplant. 2019;34(1):63-73.

28. Pan B, Jin X, Jun L, Qiu S, Zheng Q, Pan M. The relationship between smoking and stroke: a meta-analysis. Medicine (Baltimore). 2019;98(12): e14872.

29. Zhu X, Yu L, Zhou H, Ma Q, Zhou X, Lei T, et al. Atherogenic index of plasma is a novel and better biomarker associated with obesity: a population-based cross-sectional study in China. Lipids Health Dis. 2018; 17(1):37.

30. Lee MJ, Park JT, Han SH, Kim YL, Kim YS, Yang CW et al. The atherogenic index of plasma and the risk of mortality in incident dialysis patients: results 
from a nationwide prospective cohort in Korea. PLoS One. 2017;12(5): e0177499.

31. Uslu AU, Kucuk A, Icli A, Cure E, Sakiz D, Arslan S, et al. Plasma atherogenic index is an independent indicator of subclinical atherosclerosis in systemic lupus erythematosus. Eurasian J Med. 2017;49(3):193-7.

32. Cure E, Icli A, Ugur Uslu A, Aydoğan Baykara R, Sakiz D, Ozucan M, et al. Atherogenic index of plasma may be strong predictor of subclinical atherosclerosis in patients with Behçet disease. Z Rheumatol. 2017;76(3): 259-66.

33. Cure E, Icli A, Uslu AU, Sakiz D, Cure MC, Baykara RA, et al. Atherogenic index of plasma: a useful marker for subclinical atherosclerosis in ankylosing spondylitis : AIP associate with cIMT in AS. Clin Rheumatol. 2018;37(5):127380.

34. Harchaoui KE, Visser ME, Kastelein JJ, Stroes ES, Dallinga-Thie GM. Triglycerides and cardiovascular risk. Curr Cardiol Rev. 2009;5(3):216-22.

35. Hewing B, Moore KJ, Fisher EA. HDL and cardiovascular risk: time to call the plumber? Circ Res. 2012;111(9):1117-20.

36. Ference BA, Ginsberg HN, Graham I, Ray KK, Packard CJ, Bruckert E, et al. Low-density lipoproteins cause atherosclerotic cardiovascular disease. 1. Evidence from genetic, epidemiologic, and clinical studies. A consensus statement from the European Atherosclerosis Society Consensus Panel. Eur Heart J. 2017:38(32):2459-72.

37. D'Agostino RB Sr, Vasan RS, Pencina MJ, Wolf PA, Cobain M, Massaro JM et al. General cardiovascular risk profile for use in primary care: the Framingham heart study. Circulation. 2008;117(6):743-53.

38. Amaya-Amaya J, Montoya-Sánchez L, Rojas-Villarraga A. Cardiovascular involvement in autoimmune diseases. Biomed Res Int. 2014;2014:367359.

39. Nurmohamed MT. Atherogenic lipid profiles and its management in patients with rheumatoid arthritis. Vasc Health Risk Manag. 2007;3(6):845-52.

40. Yates M, Watts RA, Bajema IM, Cid MC, Crestani B, Hauser T, et al. EULAR/ ERA-EDTA recommendations for the management of ANCA-associated vasculitis. Ann Rheum Dis. 2016;75(9):1583-94.

41. Flossmann O, Bacon P, de Groot K, Jayne D, Rasmussen N, Seo P, et al. Development of comprehensive disease assessment in systemic vasculitis. Ann Rheum Dis. 2007;66(3):283-92

42. Juárez-Pérez CA, Aguilar-Madrid G, Haro-García LC, Gopar-Nieto R, CabelloLópez A, Jiménez-Ramírez C, et al. Increased cardiovascular risk using atherogenic index measurement among healthcare workers. Arch Med Res. 2015;46(3):233-9.

\section{Publisher's Note}

Springer Nature remains neutral with regard to jurisdictional claims in published maps and institutional affiliations.

Ready to submit your research? Choose BMC and benefit from:

- fast, convenient online submission

- thorough peer review by experienced researchers in your field

- rapid publication on acceptance

- support for research data, including large and complex data types

- gold Open Access which fosters wider collaboration and increased citations

- maximum visibility for your research: over $100 \mathrm{M}$ website views per year

At $\mathrm{BMC}$, research is always in progress.

Learn more biomedcentral.com/submissions 